\title{
VARIABILIDADE ESPACIAL DE PROPRIEDADES FÍSICO-HÍDRICAS DE UM NITOSSOLO SOB A CULTURA DO FEIJOEIRO IRRIGADO
}

\author{
RACHEL M. L. GUIMARÃES ${ }^{1}$, ANTÔNIO C. A. GONÇALVES ${ }^{2}$, CÁSSIO A. TORMENA ${ }^{3}$, \\ MARCOS V. FOLEGATTI ${ }^{4}$, ÉVERTON BLAINSKI ${ }^{5}$
}

\begin{abstract}
RESUMO: Devido à utilização mais intensa de água via irrigação e ao elevado custo que tem sido agregado a este recurso, é necessário que os sistemas de irrigação operem com altos níveis de eficiência. A descrição do padrão espacial de propriedades físico-hídricas do solo pode auxiliar no manejo eficiente da irrigação. Para tanto, técnicas da geoestatística são necessárias. Assim, este trabalho teve como objetivos descrever e avaliar o padrão espacial da porosidade, densidade e armazenamento de água no solo durante um ciclo de secamento, num Nitossolo Vermelho distroférrico cultivado com a cultura do feijoeiro. A área de cultivo foi subdividida em 80 quadrículas, segundo uma malha de 3 por $3 \mathrm{~m}$. Em cada quadrícula, mediu-se o teor de água do solo ao longo do tempo, com sondas de TDR de 0,20 m de comprimento, e coletou-se uma amostra de solo indeformada. Os dados foram analisados por meio da estatística descritiva, análise exploratória e técnicas da geoestatística. A porosidade total, a macroporosidade e a microporosidade ocorreram de forma aleatória na área. A densidade do solo apresentou moderado grau de dependência espacial. Verificou-se que a uniformidade de aplicação da lâmina de irrigação não alterou o padrão espacial de armazenamento de água no solo, evidenciando que fatores intrínsecos do solo definiram este padrão.
\end{abstract}

PALAVRAS-CHAVE: distribuição espacial, geoestatística, armazenamento de água no solo, porosidade do solo, densidade do solo.

\section{SPATIAL DISTRIBUTION OF PHYSICAL-HYDRIC PROPERTIES OF A NITOSOLS UNDER IRRIGATED BEAN CROP}

\begin{abstract}
Due to the intensive utilization of water for irrigation and the high costs of this resource, irrigation systems need to run with high levels of efficiency. The spatial pattern of soil properties can help to improve the efficiency of irrigation management. For this, geostatistic techniques are needed. The objectives of this work were to describe statistically and to evaluate the spatial variability of bulk density, soil porosity and water storage, on a Red Nitosols, cultivated with irrigated beans. The experimental area was sub-divided into 80 squares, in a $3 \mathrm{~m}$ squared grid. On each square measurements of soil moisture during the dry cycle, with $0.20 \mathrm{~m}$ of depth TDR probes and an undisturbed soil sample were taken. Data was analyzed by descriptive statistics, exploratory analysis and geostatistic techniques. It was found that the total porosity, macroporosity and microporosity are randomly distributed variables. Soil bulk density presented a moderated level of spatial dependence. The uniformity of the amount of water applied does not influence the water storage pattern, but intrinsic soil factors are determining factors.
\end{abstract}

KEYWORDS: spatial distribution, geostatistic, soil water storage, soil porosity, soil bulk density.

\footnotetext{
${ }^{1}$ Eng ${ }^{\text {a }}$ Agrônoma, Doutoranda, Programa de Pós-Graduação em Agronomia, Universidade Estadual de Maringá - PR, rachellocks@gmail.com.

${ }^{2}$ Professor Adjunto, Departamento de Agronomia, UEM, Maringá - PR.

${ }^{3}$ Professor Associado do Departamento de Agronomia, UEM, Maringá - PR.

${ }^{4}$ Professor Titular, Departamento de Engenharia Rural, Escola Superior de Agricultura "Luiz de Queiroz", USP, Piracicaba - SP.

${ }^{5}$ Eng $^{\mathrm{o}}$ Agrônomo, Doutor em Agronomia, Pesquisador da Empresa de Pesquisa Agropecuária e Extensão Rural de Santa Catarina EPAGRI.

Recebido pelo Conselho Editorial em: 5-5-2008
}

Aprovado pelo Conselho Editorial em: 28-5-2010 


\section{INTRODUÇÃO}

Com o crescente uso da irrigação e a competição pela utilização da água, é necessário que os sistemas de irrigação operem com elevados níveis de uniformidade e de eficiência de aplicação. Assim, os parâmetros que expressam a qualidade da irrigação devem ser entendidos como componentes decisórios do planejamento e operação destes sistemas (FRIZZONE, 1992). Além disso, reconhece-se que a uniformidade de aplicação de água é diretamente relacionada com o rendimento da cultura (GOHRING \& WALLENDER, 1987; LETEY et al., 1984; SOLOMON, 1984), apesar de que FRIZZONE et al. (2007) concluíram que a máxima uniformidade de aplicação não corresponde ao máximo rendimento da cultura do feijoeiro.

A distribuição espacial da lâmina de água aplicada via irrigação pode ter forte influência sobre o padrão espacial de armazenamento de água no solo (ARM). Entretanto, fatores intrínsecos do solo, tais como fatores e processos de formação do solo (CARVALHO et al., 2003) podem afetar a capacidade de ARM bem como a distribuição espacial destes valores (GONÇALVES et al., 1999; GONÇALVES \& FOLEGATTI, 2002). O padrão de ARM apresenta forte estabilidade temporal, sendo pouco influenciado pelo padrão espacial de lâmina aplicada (REZENDE et al., 2002 e GONÇALVES et al., 2004).

A medida do teor de água do solo em diversos pontos e momentos ao longo dos ciclos de secagem e umedecimento pode servir como base para a compreensão dos processos dinâmicos da água em escalas espaciais e temporais no sistema solo-planta (GONÇALVES et al., 1999). Para tanto, estas informações devem ser analisadas de forma a gerar conhecimento acerca destes processos, a exemplo do uso de técnicas estatísticas para o tratamento de dados espacialmente referenciados (ISAAKS \& SRIVASTAVA, 1989).

O uso de técnicas geoestatísticas constitui-se numa importante estratégia no tratamento destas informações, por permitir a descrição da distribuição espacial de uma variável qualquer, a partir da modelagem da continuidade espacial (JOURNEL \& HUIJBREGTS, 1978), bem como a avaliação da persistência no tempo dos possíveis padrões espaciais de distribuição de água no solo (KACHANOSKI \& DE JONG, 1988; GONÇALVES \& FOLEGATTI, 2002).

Neste contexto, o objetivo deste trabalho foi descrever e avaliar o padrão espacial da porosidade total $(\mathrm{Pt})$, macroporosidade (MAC), microporosidade (MIC), densidade do solo (Ds) e do armazenamento de água no solo (ARM) durante um ciclo de secamento, em um Nitossolo Vermelho distroférrico cultivado com a cultura do feijoeiro irrigado, sob preparo convencional.

\section{MATERIAL E MÉTODOS}

O trabalho foi conduzido em área experimental da Universidade Estadual de Maringá, Paraná, localizada no município de Maringá ( $\left.23^{\circ} 25^{\prime} \mathrm{S} 51^{\circ} 57^{\prime} \mathrm{W}\right)$, com altitude média de $540 \mathrm{~m}$. O clima da região é caracterizado como Cfa, de acordo com a classificação de Köeppen (PEEL et al., 2007), com chuvas abundantes no verão e inverno seco e com precipitação média anual de $1.500 \mathrm{~mm}$ (IAPAR, 2000). A temperatura média anual está entre 21 e $22^{\circ} \mathrm{C}$. O solo é classificado como Nitossolo Vermelho distroférrico, A moderado, textura argilosa (EMBRAPA, 2006). A análise granulométrica do solo foi realizada conforme métodos descritos em EMBRAPA (1997), e os teores de argila, silte e areia na camada de 0-0,15 m foram, respectivamente, de $735 \mathrm{~g} \mathrm{~kg}^{-1}, 90 \mathrm{~g} \mathrm{~kg}^{-1} \mathrm{e}$ $175 \mathrm{~g} \mathrm{~kg}^{-1}$.

A área foi cultivada com a cultura do feijoeiro (Phaseolus vulgaris L., cv. IAPAR 81, ciclo médio de 92 dias), com espaçamento entre linhas de $0,5 \mathrm{~m}$ e 15 sementes por metro. Esta área esteve em pousio com vegetação espontânea entre os anos de 2000 e 2004. Em 2004, cultivou-se a cultura do feijoeiro na safra de verão, sucedida pelo cultivo da mesma cultura, na safra de verão e inverno, do ano de 2005.

Em 2006, a cultura do feijoeiro foi cultivada na safra de inverno, para a instalação deste experimento. Para o plantio, a área foi escarificada por meio de um escarificador com cinco hastes 
estreitas espaçadas de $0,25 \mathrm{~m}$ a uma profundidade média de $0,25 \mathrm{~m}$, seguida de duas gradagens com intervalo de dez dias.

Após a segunda gradagem, foi incorporada trifuralina no solo, na dosagem de 2,4 L ha-1. Antes do plantio, a área foi adubada com $300 \mathrm{~kg} \mathrm{ha}^{-1}$ da formulação 4-20-20 de NPK, como o auxílio de uma semeadora de 5 linhas, de acordo com análises do solo. O plantio foi feito manualmente com o uso de matracas. Durante o ciclo da cultura, foram realizadas três capinas manuais, três adubações de cobertura com sulfato de amônio, totalizando $100 \mathrm{~kg}$ de Nitrogênio por hectare, e duas aplicações de inseticida para o controle de Diabrotica speciosa.

O sistema de irrigação por aspersão convencional foi instalado com espaçamento entre aspersores de 15 x 18 m (Figura 1), os quais apresentavam diâmetro dos bocais de 5,6 x 3,2 mm. Nesta configuração, operando com pressão de $35 \mathrm{mca}$, a vazão aplicada pelo aspersor foi de $2,90 \mathrm{~m}^{3} \mathrm{~h}^{-1}$, em um círculo irrigado com $32 \mathrm{~m}$ de diâmetro. O espaçamento utilizado permitiu a aplicação de uma lâmina de água cujo coeficiente de uniformidade de Christiansen (CUC) foi de $80 \%$, em média.

A área selecionada entre dois terraços foi subdividida em 80 quadrículas (pontos experimentais), formando uma malha de pontos espaçados de 3 por $3 \mathrm{~m}$, totalizando uma área de $48 \times 15 \mathrm{~m}\left(720 \mathrm{~m}^{2}\right)$. No centro de cada quadrícula, instalou-se uma sonda de TDR na camada de 0-0,20 m, para monitoramento do teor de água do solo para o fim de manejo da irrigação e coleta de dados para este experimento. As sondas utilizadas foram fabricadas e calibradas conforme metodologia descrita por TRINTINALHA (2005). Ao lado de cada sonda, foi instalado um pluviômetro para coleta de lâmina de água de chuva e irrigação. Na Figura 1, é mostrado o croqui da área experimental.

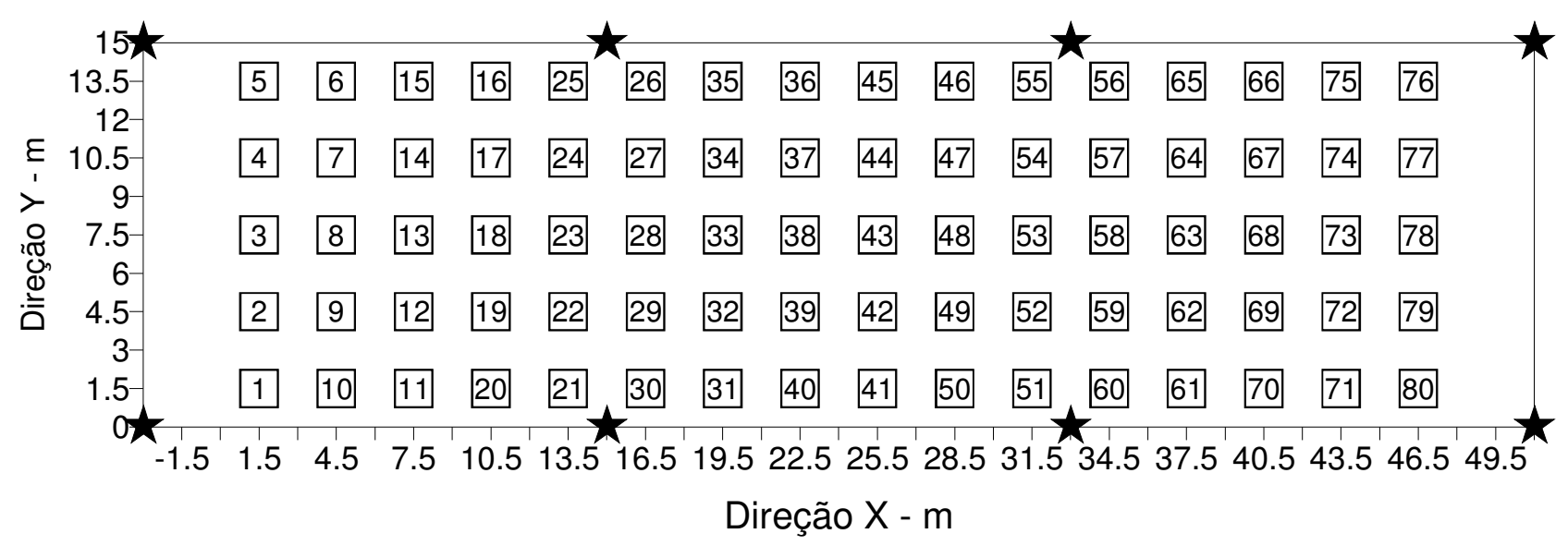

FIGURA 1. Croqui da área experimental. As estrelas representam os aspersores dispostos no espaçamento 15 x $18 \mathrm{~m}$. Os quadrados representam o centro das quadrículas, apresentando a ordem dos pontos amostrados. . Scheme of the experimental area. The stars represent sprinklers placed on a spacing of 15 x $18 \mathrm{~m}$. The squares represent the centre of the grids, showing the order of the sampled points.

Durante o ciclo da cultura, foram realizadas dezesseis irrigações. Cada irrigação foi conduzida de forma a elevar o teor de água do solo até um valor próximo à capacidade de campo $\left(0,47 \mathrm{~m}^{3} \mathrm{~m}^{-3}\right)$. De um modo geral, as irrigações foram realizadas quando o déficit de água no solo, na camada de $0-0,20 \mathrm{~m}$, atingia valores da ordem de $15 \mathrm{~mm}$.

Considerando que o padrão de aplicação de água de irrigação foi sempre o mesmo, selecionou-se um ciclo de secamento do solo após a irrigação realizada no dia 172 do ano de 2006 para a análise da distribuição espacial do ARM. Nesse período, a cultura encontrava-se na fase de formação de vagens. O teor de água do solo foi monitorado periodicamente até o dia 176, quando o seu valor médio na camada de $0-0,20 \mathrm{~m}$ se apresentava próximo ao ponto de murcha permanente 
$\left(0,33 \mathrm{~m}^{3} \mathrm{~m}^{-3}\right)$. A medida do teor de água do solo foi realizada antes da irrigação (AA) e imediatamente após a irrigação (A1). A irrigação foi realizada no dia 176, ao final de tarde, quando a velocidade do vento usualmente se reduz.

Durante a aplicação de água, pôde-se verificar alguma alteração da velocidade do vento, como é comum ocorrer, o que promoveu alguma irregularidade na aplicação de água pelos aspersores. Como o propósito do trabalho é a avaliação da distribuição de água no solo, a partir da aplicação de água via irrigação, esta irregularidade gerada pelo vento foi entendida como favorável ao estudo. Admitiu-se evaporação nula durante a irrigação, a partir da leitura de lâmina de água em três coletores dispostos ao lado da área irrigada.

Estas leituras foram realizadas no início e no final da irrigação, após a adição de cerca de $100 \mathrm{~mL}$ de água em cada coletor. A lâmina de água aplicada nesta irrigação foi coletada a fim de estudar a sua relação com o ARM. Em seguida, foram feitas medidas de teor de água do solo em 26; 48; 72 e 98 horas após a irrigação. O ARM foi calculado por meio da multiplicação do teor de água do solo pela profundidade de $0,20 \mathrm{~m}$.

Após a colheita, no centro de cada quadrícula, foram coletadas amostras indeformadas de solo na camada de 0-0,10 m, por meio de anéis com 0,05 $\mathrm{m}$ de diâmetro e altura. Com o auxílio de um atuador hidráulico, acoplado em um trator, os anéis foram introduzidos no solo lentamente, com o objetivo de evitar ao máximo a compactação da amostra. As amostras indeformadas foram envolvidas com papel alumínio até o preparo das mesmas em laboratório.

Após devidamente preparadas, estas amostras foram saturadas por 48 horas numa bandeja com água até dois terços da altura dos anéis. Obteve-se a massa do solo saturado e em seguida, as amostras foram drenadas na tensão equivalente a $-60 \mathrm{hPa}(\mathrm{hPa}$ igual a cm de coluna de água), utilizando-se de uma mesa de tensão adaptada de KHIEL (1979). A partir dos valores de retenção de água das amostras, calculou-se o volume de macroporos drenados na tensão de $-60 \mathrm{hPa}$ (MAC), a MIC e a Pt, de acordo com EMBRAPA (1997). A Ds foi determinada de acordo com BLAKE \& HARTGE (1986).

A análise descritiva e exploratória dos dados foi realizada utilizando-se do software Statistica (STATSOFT, 1993), conforme ISAAKS \& SRIVASTAVA (1989) para verificar o atendimento à hipótese de estacionaridade, ao menos intrínseca, do conjunto de dados. Após esta etapa, atendendo às hipóteses necessárias, foram construídos semivariogramas experimentais para descrever a estrutura de dependência espacial de cada variável, utilizando-se do software Geoeas (ENGLUND \& SPARKS, 1988) e Variowin (PANNATIER, 1996).

Utilizando a técnica da krigagem (VIEIRA et al., 1983) foram construídas as superfícies que mostram os valores de cada uma das variáveis medidas, nas respectivas posições no espaço. A interpolação foi realizada a partir dos valores medidos experimentalmente, utilizando-se o modelo ajustado ao semivariograma experimental. As análises de regressão foram obtidas entre os valores dos 80 pontos, e os coeficientes lineares foram submetidos ao teste t, adotando-se $\alpha=5 \%$ (SAS, 1999).

\section{RESULTADOS E DISCUSSÃO}

Os momentos estatísticos para Ds, Pt, MAC, MIC, lâmina de irrigação aplicada e armazenamento de água no solo antes da irrigação (AA), após a irrigação (A1) e no período de 26 (A26), 48 (A48), 72 (A72) e 98 (A98) horas após a irrigação são mostrados na Tabela 1. 
TABELA 1. Momentos estatísticos de densidade do solo (Ds), porosidade total (Pt), macroporosidade (MAC), microporosidade (MIC), lâmina aplicada (Lam) e dos valores de armazenamento de água no solo medidos nos diferentes momentos $\left(\mathrm{A}_{\mathrm{i}}\right)$.

Statistical moments of soil bulk density (Ds), total porosity (Pt), macroporosity (MAC), microporosity (MIC), applied water (Lam) and water storage values measured at different times $(\mathrm{Ai})$.

\begin{tabular}{|c|c|c|c|c|c|c|c|}
\hline & \multicolumn{2}{|c|}{$\mathrm{Ds}^{-\mathrm{Mg} \mathrm{m}^{-3}}$} & \multicolumn{2}{|c|}{ Pt- $m^{3} m^{-3}$} & MAC- $\mathrm{m}^{3} \mathrm{~m}^{-3}$ & \multicolumn{2}{|c|}{ MIC- $\mathrm{m}^{3} \mathrm{~m}^{-3}$} \\
\hline Média & \multicolumn{2}{|c|}{1,36} & \multicolumn{2}{|l|}{0,57} & 0,10 & \multicolumn{2}{|c|}{0,47} \\
\hline Mediana & \multicolumn{2}{|c|}{1,37} & \multicolumn{2}{|l|}{0,57} & 0,10 & \multicolumn{2}{|c|}{0,47} \\
\hline $\mathrm{d}\left(\mathrm{K}-\mathrm{S}^{1}\right)$ & \multicolumn{2}{|c|}{$0,11 *$} & \multicolumn{2}{|l|}{$0,11 *$} & $0,10 *$ & \multicolumn{2}{|c|}{$0,07 *$} \\
\hline Mínimo & \multicolumn{2}{|c|}{1,11} & \multicolumn{2}{|l|}{0,54} & 0,05 & \multicolumn{2}{|c|}{0,41} \\
\hline Máximo & \multicolumn{2}{|c|}{1,47} & \multicolumn{2}{|l|}{0,65} & 0,22 & \multicolumn{2}{|c|}{0,50} \\
\hline Q. inferior ${ }^{2}$ & \multicolumn{2}{|c|}{1,32} & \multicolumn{2}{|l|}{0,56} & 0,08 & \multicolumn{2}{|c|}{0,46} \\
\hline Q. superior $^{2}$ & \multicolumn{2}{|c|}{1,41} & \multicolumn{2}{|l|}{0,58} & 0,12 & \multicolumn{2}{|c|}{0,48} \\
\hline A. interq. ${ }^{3}$ & \multicolumn{2}{|c|}{0,08} & \multicolumn{2}{|l|}{0,03} & 0,04 & \multicolumn{2}{|c|}{0,02} \\
\hline D.-padrão ${ }^{4}$ & \multicolumn{2}{|c|}{0,07} & \multicolumn{2}{|l|}{0,02} & 0,04 & \multicolumn{2}{|c|}{0,02} \\
\hline Assimetria & $-1,0$ & & 1,05 & & 1,03 & & \\
\hline Curtose & 1,3 & & 1,38 & & 1,22 & & \\
\hline C.V..$^{5}$ & 5,1 & & 3,8 & & 33,8 & & \\
\hline L. inferior ${ }^{6}$ & 1,2 & & 0,52 & & 0,01 & & \\
\hline L. superior ${ }^{6}$ & 1,5 & & 0,62 & & 0,19 & & \\
\hline & & & Variáve & $\mathrm{s}-\mathrm{mm}$ & & & \\
\hline & Lam & AA & A1 & A 26 & A 48 & A 72 & A 98 \\
\hline Média & 17,58 & 67,44 & 81,79 & 77,54 & 75,70 & 73,30 & 69,53 \\
\hline Mediana & 18,49 & 67,71 & 80,56 & 77,74 & 75,72 & 73,16 & 69,82 \\
\hline$d\left(K-S^{1}\right)$ & $0,14^{*}$ & $0,07 *$ & $0,07 *$ & $0,10^{*}$ & $0,09 *$ & $0,09 *$ & $0,11^{*}$ \\
\hline Mínimo & 7,87 & 50,99 & 57,68 & 56,97 & 56,62 & 53,80 & 49,93 \\
\hline Máximo & 25,30 & 80,20 & 107,31 & 102,38 & 98,51 & 93,58 & 87,24 \\
\hline Q. inferior ${ }^{2}$ & 15,77 & 64,36 & 76,51 & 74,22 & 71,05 & 69,64 & 66,12 \\
\hline Q. superior ${ }^{2}$ & 20,23 & 71,05 & 86,54 & 80,73 & 78,88 & 76,95 & 73,34 \\
\hline A. interq. ${ }^{3}$ & 4,46 & 6,69 & 10,03 & 6,51 & 7,83 & 7,30 & 7,22 \\
\hline D.-padrão ${ }^{4}$ & 4,11 & 5,17 & 9,15 & 7,32 & 6,82 & 6,38 & 5,94 \\
\hline Assimetria & $-0,76$ & $-0,41$ & 0,21 & 0,26 & 0,26 & $-0,03$ & $-0,37$ \\
\hline Curtose & 0,17 & 0,80 & 0,73 & 1,80 & 1,60 & 1,42 & 1,55 \\
\hline C.V. ${ }^{5}$ & 23,4 & 7,6 & 11,1 & 9,4 & 9,0 & 8,7 & 8,5 \\
\hline L. inferior ${ }^{6}$ & 9,08 & 54,33 & 61,46 & 64,45 & 59,30 & 58,69 & 55,30 \\
\hline L. superior ${ }^{6}$ & 26,92 & 81,08 & 101,59 & 90,50 & 90,63 & 87,90 & 84,16 \\
\hline
\end{tabular}

*- distribuição normal para $\alpha=5 \%$ de probabilidade / d crítico a 5\%: 0,15 (CAMPOS, 1983). ${ }^{1}$ Kolmogorov-Smirnov. ${ }^{2}$ Quartil inferior e superior. ${ }^{3}$ Amplitude interquartílica. ${ }^{4}$ Desvio-padrão. ${ }^{5}$ Coeficiente de variação (\%). ${ }^{6}$ Limites inferior e superior para candidatos a "outliers", segundo metodologia empregada por LIBARDI et al. (1996).

A semelhança entre os valores de média e mediana indica a simetria da distribuição dos dados para todas as propriedades, em conformidade com COSTA NETO (1990). De acordo com BEIGUELMAN (1994), quanto à simetria das distribuições, pode-se considerá-las simétricas e mesocúrticas $(P<0,05)$. Isto é confirmado pelo teste de Kolmogorov-Smirnov (d). Para uma distribuição normal, os coeficientes de assimetria e curtose devem assumir valor zero, mas conforme mostrado na Tabela 1, os valores destes coeficientes apresentam-se ligeiramente diferentes de zero. No entanto, não caracterizam afastamento expressivo da normalidade, apenas evidenciam que, conforme salientado por NIELSEN et al. (1973), na natureza, não são encontradas distribuições que sejam absolutamente normais. A normalidade para MAC também foi verificada por DA MATA et al. (1998) e SOUZA et al. (2004), e Ds por COELHO FILHO et al. (2001), SOUZA et al. (2004), CAVALIERI et al. (2006), FIDALSKI et al. (2006) e FIDALSKI \& 
TORMENA (2007). O valor elevado do coeficiente de variação $(33,8 \%)$ para MAC deve-se à ampla variação da densidade do solo, propiciando a ocorrência de elevada dispersão da MAC, que variou entre 0,05 a $0,22 \mathrm{~m}^{3} \mathrm{~m}^{-3}$. Além disso, a média igual a $0,10 \mathrm{~m}^{3} \mathrm{~m}^{-3}$, ou seja, relativamente próxima de zero, tende a fazer com que o coeficiente de variação (C.V.) assuma valores mais elevados. Na média, pode-se verificar que a aeração do solo se encontra em boas condições, $\mathrm{MAC}=0,10 \mathrm{~m}^{3} \mathrm{~m}^{3}$, valor considerado satisfatório para que as plantas tenham adequado desenvolvimento radicular (GRABLE \& SIEMER, 1968; DEXTER, 1988; SILVA et al., 1994; TORMENA et al., 2007; BLAINSKI et al., 2009). Entretanto, cerca de $45 \%$ da área encontram-se com $M A C<0,10 \mathrm{~m}^{3} \mathrm{~m}^{3}$, apontando para a degradação física do solo (Figura 2a). Estes resultados permitem a constatação da importância de se estudarem as propriedades físicas do solo espacialmente, uma vez que, na média, a aeração é considerada satisfatória. Porém, praticamente metade da área apresenta valores inferiores ao considerado adequado. Verificou-se correlação inversa entre MAC e Ds (Figura 2b). Para Ds $>1,37 \mathrm{Mg} \mathrm{m}^{-3}$, a MAC torna-se inferior a $10 \%$, ou seja, nesta condição, a aeração do solo torna-se deficiente, expondo a cultura a uma condição de estresse (Figura 2b). Portanto, práticas de manejo do solo que visem à redução da Ds deveriam ser adotadas com vistas no controle da MAC. Em áreas irrigadas, o manejo da irrigação é realizado a fim de elevar o potencial de água no solo para valores próximos à capacidade de campo. Este procedimento, conduzido na área em estudo, poderia acarretar danos consideráveis à cultura, com relação à aeração, em decorrência do fato de que grande parte da área apresenta valores de densidade acima de $1,37 \mathrm{Mg} \mathrm{m}^{-3}$.
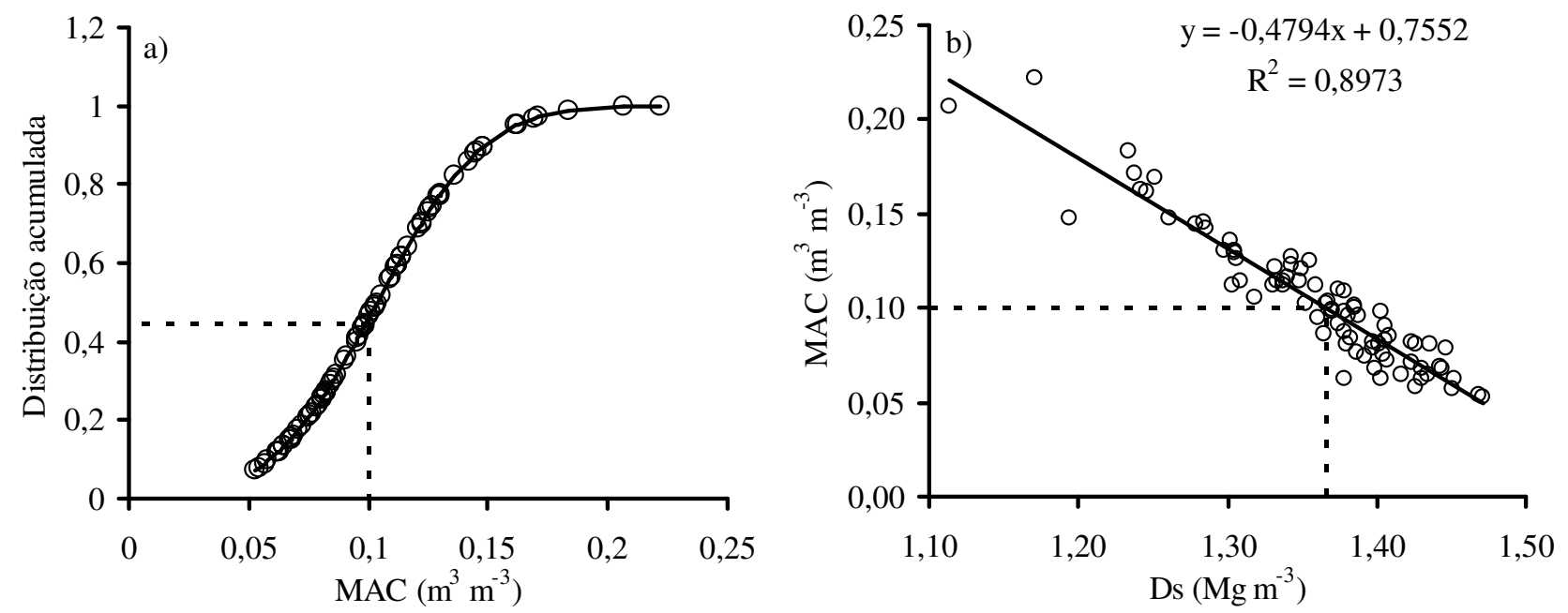

FIGURA 2. Distribuição acumulada de macroporosidade (MAC). As linhas pontilhadas referem-se à distribuição em que MAC $=0,10 \mathrm{~m}^{3} \mathrm{~m}^{-3}$ (a). Macroporosidade (MAC) em função da Densidade do solo (Ds). As linhas pontilhadas referem-se à Ds em que MAC = $0,10 \mathrm{~m}^{3} \mathrm{~m}^{-3}$ (b). Accumulated distribution of macroporosity (MAC). The broken lines refer to $\mathrm{MAC}=0,10 \mathrm{~m}^{3} \mathrm{~m}^{-3}$ (a). Macroporosity (MAC) as a function of soil bulk density (Ds). The broken lines refer to Ds where MAC $=0,10 \mathrm{~m}^{3} \mathrm{~m}^{-3}(\mathrm{~b})$.

As distribuições de ARM apresentaram CV classificado como baixo, segundo WARRICK \& NIELSEN, (1980), assumindo valores entre 7 e 11\% (Tabela 1). A estatística d, do teste de Kolmogorov-Smirnov, indicou que todas as distribuições de ARM apresentaram distribuição normal, em conformidade com ROCHA et al. (2005) e TRINTINHALHA (2005). Os coeficientes de assimetria para ARM assumiram valores próximos de zero (Tabela 1), evidenciando a simetria das distribuições, e corroboraram a aceitação da hipótese de normalidade apontada pelo teste de Kolmogorov-Smirnov. A lâmina de irrigação aplicada apresentou grande amplitude total (Tabela 1), caracterizando uma aplicação de água desuniforme na área com C.V.=23,4\%, constituindo numa variação classificada como média, segundo WARRICK e NIELSEN (1980). Destaca-se, no entanto, que o coeficiente de uniformidade de Christiansen (CUC) para esta irrigação assumiu o valor de $78 \%$, próximo ao valor tradicionalmente adotado de $80 \%$, que é considerado aceitável para a irrigação por aspersão (CHRISTIANSEN, 1942). Conforme descrito, essa pequena redução de valor 
está associada à variação da velocidade do vento, durante a aplicação de água. A assimetria negativa da distribuição dos valores de lâmina de irrigação aplicada permite verificar que existe pequena concentração de valores para a esquerda da distribuição, caracterizada também pela existência de valores candidatos a "outliers" abaixo do limite inferior. O valor aproximado de zero para o coeficiente de curtose evidencia que a concentração de valores em torno da média é compatível com a normalidade, contribuindo para a aceitação da hipótese de normalidade, apontada pelo teste de Kolmogorov-Smirnov. Resultado similar foi obtido por PAZ et al. (2002).

A distribuição espacial dos valores de lâmina de água aplicada e de ARM na camada de 0,20 m é mostrada na Figura 3. Pode-se verificar que o solo apresentava antes da irrigação (AA) um padrão de distribuição dos valores de ARM com certa dispersão, caracterizada por um C.V. de 7,66\% (Tabela 1). A aplicação de água via irrigação, apesar de desuniforme (C.V. de 23,4\%), não determinou mudanças no padrão de distribuição de ARM. Embora em alguns pontos ocorra uma variação de ARM relativamente grande antes da irrigação (AA) e após a irrigação (A1), decorrente de maiores valores de lâmina aplicada, o padrão de distribuição espacial foi pouco alterado.

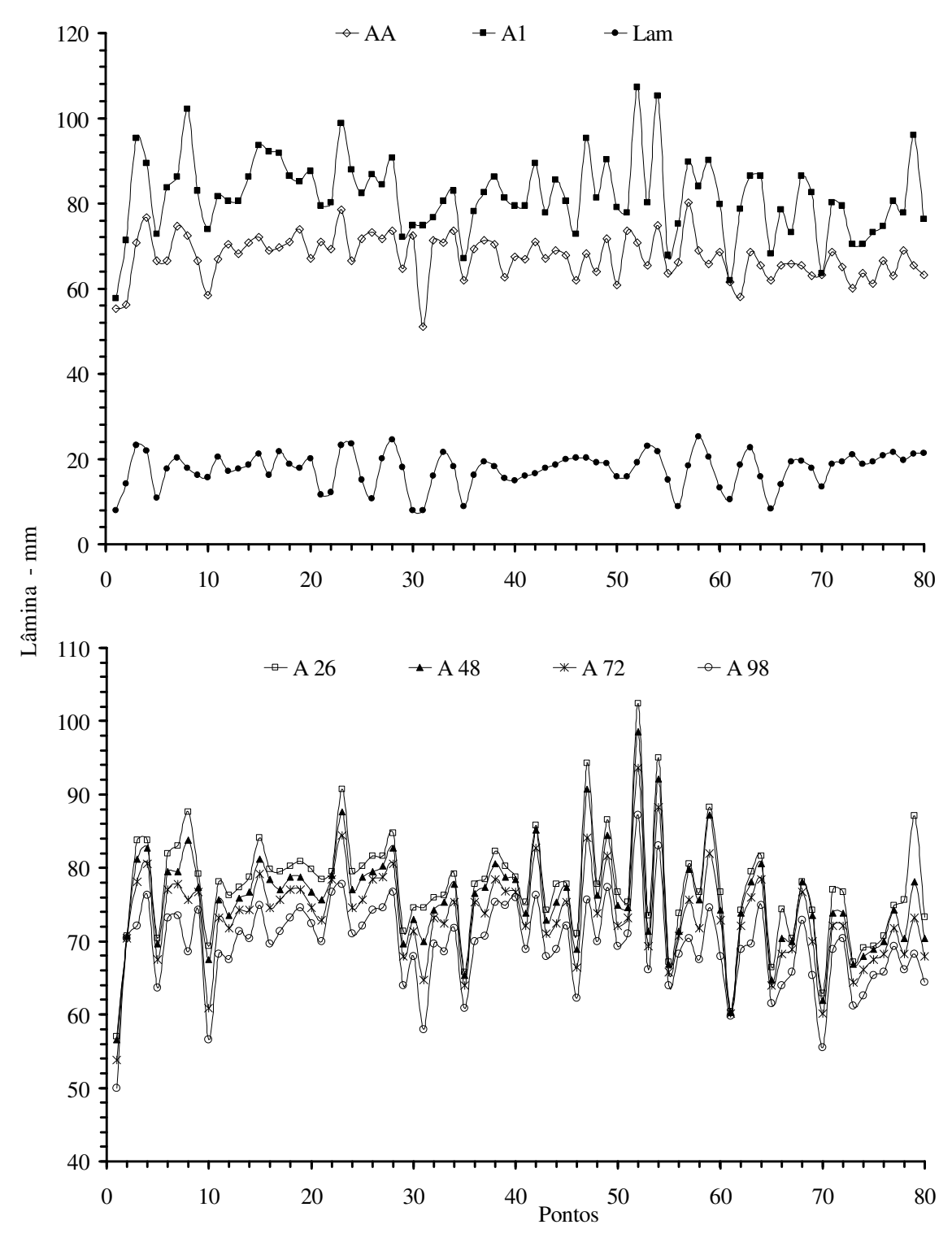

FIGURA 3. Valores de lâmina de água de irrigação aplicada (Lam) e de armazenamento de água no solo, em mm, antes da aplicação de água (AA), após a irrigação (A1) e em diferentes momentos após a irrigação (A26, A48, A72, A98). Values of applied water (Lam) and soil water storage, $\mathrm{mm}$, before irrigation (AA), after irrigation (A1) and in different times after irrigation (A26, A48, A72, A98). 
Após a aplicação de água, a baixa uniformidade da aplicação impôs certa dispersão nos valores de ARM, verificado pelo aumento do CV para 11,19\% (A1). Porém, com o tempo, verificou-se que a dispersão diminuiu, havendo uma tendência para retornar ao padrão inicial. Como pode ser visto na Tabela 1, o CV de A26 é de 9,4\%, que diminui progressivamente para 9,0; 8,7 e 8,5\% em A48, A72 e A98, respectivamente, tendendo à uniformização do ARM. A redistribuição e o consumo de água pelas plantas tendem a uniformizar o conteúdo de água no solo, além de estarem relacionados com a variabilidade espacial intrínseca do solo (REZENDE et al., 2002).

A análise espacial revelou que MAC, MIC e Pt não apresentaram estrutura de dependência espacial. Resultado semelhante foi verificado por BERNER et al. (2007) em um solo sem cultivo. Entretanto, pode-se verificar, em trabalhos como de SOUZA et al. (2004), que existe estrutura de dependência espacial para essas variáveis. A ausência de estrutura de dependência espacial de Pt, MAC e MIC pode ser associada à grande movimentação que ocorre na camada amostrada (0-0,10 m), promovida pelo preparo convencional do solo, principalmente pela ação de gradagem, fazendo com que a porosidade do solo ocorra de maneira heterogênea e aleatória no espaço (DA MATA et al., 1998). A densidade do solo apresentou semivariograma experimental com grau de estrutura de dependência espacial (GD) moderado $\left(\mathrm{C}_{0}=0,7 ; \mathrm{C}_{0}+\mathrm{C}=1,04 ; \mathrm{a}=9 \mathrm{~m}\right)$, segundo classificação de CAMBARDELLA et al. (1994). Resultado similar foi verificado por SOUZA et al. (2004). Por outro lado, FIDALSKI et al. (2006) não encontraram estrutura de dependência espacial para a densidade do solo.

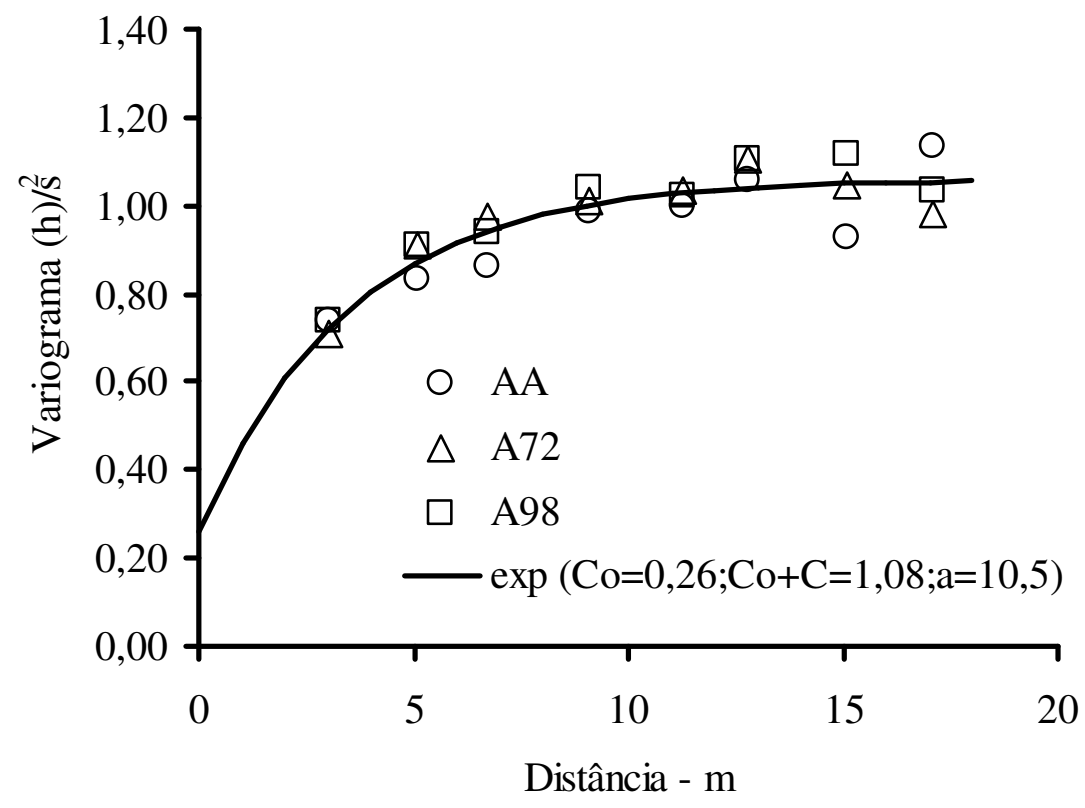

FIGURA 4. Semivariograma experimental escalonado do armazenamento de água no solo antes da irrigação (AA), e 72 horas (A72) e 98 horas (A98) após a irrigação. Scaled experimental semivariogram of soil water storage, before irrigation (AA), 72 hours (A72) and 98 hours (A98) after irrigation.

Apenas AA, A72 e A98 apresentaram estrutura de dependência espacial. Os semivariogramas experimentais escalonados pela variância amostral mostraram a existência de uma forte estrutura de dependência espacial, GD=24,53\% (CAMBARDELLA et al., 1994), descrita por meio de um modelo exponencial cujo alcance foi de 10,5 m (Figura 4). Esses resultados corroboram aqueles de TRINTINALHA (2005), que identificou uma forte estrutura de dependência espacial, descrita com modelo exponencial, com elevada persistência no tempo para essa variável, na mesma área experimental, bem como os de GONÇALVES et al. (1999).

O fato de A1, A26 e A48 não terem apresentado estrutura de dependência espacial pode estar relacionada com a redistribuição de água no solo, que ocorre após a aplicação de água. De acordo 
com FRIZZONE et al. (2007), a aplicação desuniforme de água no solo cria gradientes de potencial que provocam o movimento da água de pontos com maior potencial para pontos de menor potencial, de modo que esse movimento faz com que o processo de redistribuição de água no solo ocorra de maneira aleatória. Além disto, a distribuição aleatória dos poros de maior diâmetro na área, responsável pela drenagem da água, e a distribuição aleatória de poros de menor diâmetro, responsáveis pela redistribuição de água no solo, também estabelecem o controle deste processo e contribuem para que, nestes momentos (A1, A26 e A48), o ARM estivesse ocorrendo de maneira aleatória no espaço. A partir das medidas realizadas 72 horas após a aplicação da água (A72), o ARM apresentou estrutura de dependência espacial. De acordo com GONÇALVES et al. (1999) e REZENDE et al. (2002), nessas condições, o ARM é ditado fundamentalmente por fatores intrínsecos do solo, resultante da variabilidade associado aos fatores e processos de formação do solo e da ação de processos hidrológicos.

A distribuição do ARM pôde ser descrita procedendo-se à interpolação por krigagem, a partir dos valores medidos, utilizando-se do modelo ajustado aos semivariogramas experimentais escalonados (Figura 5).

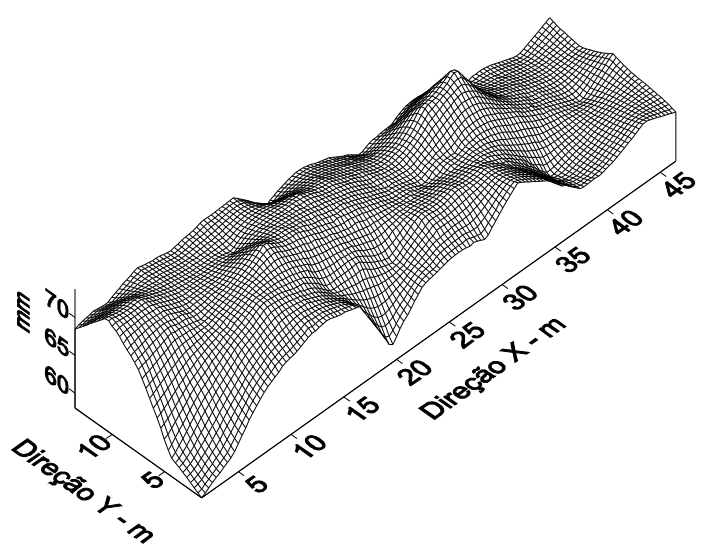

(a)

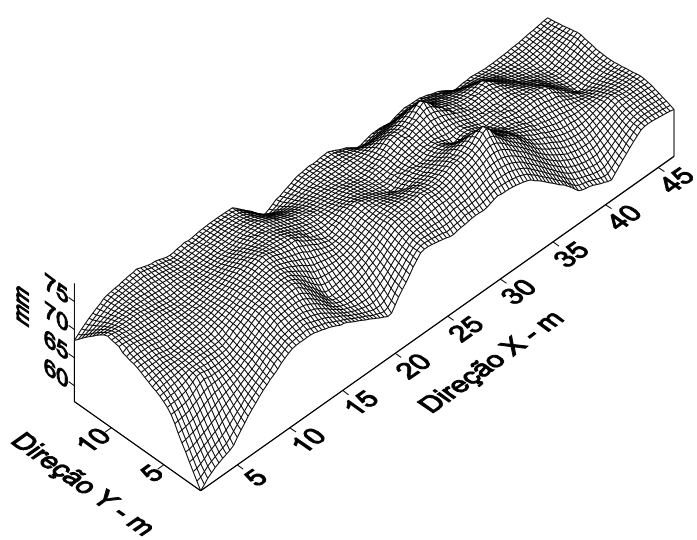

(c)

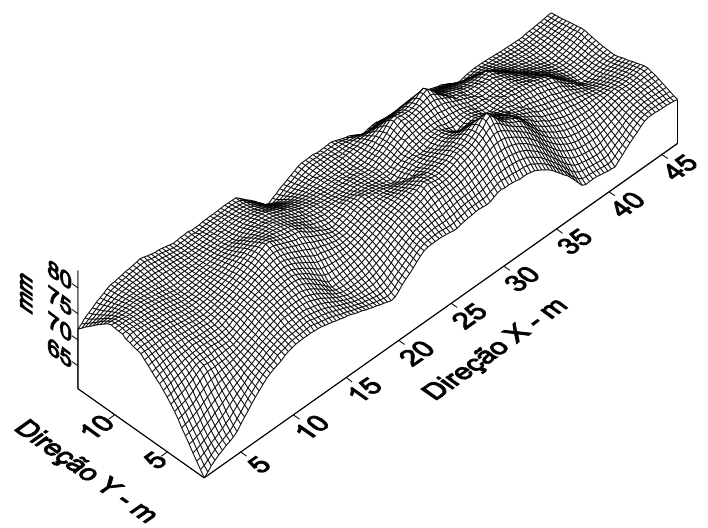

(b)

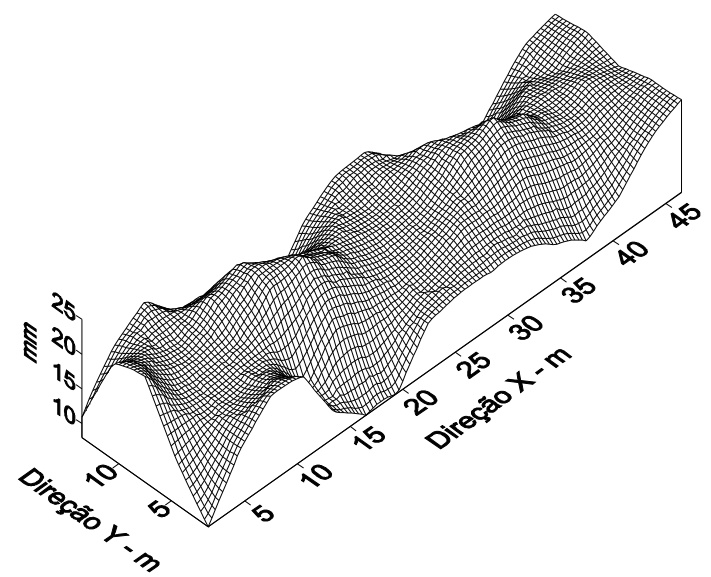

(d)

FIGURA 5. Distribuição espacial dos valores de armazenamento antes da irrigação (a), após 72 horas (b), após 98 horas (c), e lâmina de irrigação aplicada (d). Spatial distribution of water storage before irrigation (a), after 72 hours (b), after 98 hours, and water applied at the irrigation event $(d)$.

Pode-se verificar, na Figura 5d, que os valores das maiores lâminas aplicadas concentraram-se ao longo da porção intermediária da área. Portanto, os valores menores de lâmina estão concentrados nas extremidades correspondentes aos valores extremos de Y. A Figura 5a apresenta a distribuição do armazenamento de água no solo antes da irrigação. Embora possa ser observada 
uma considerável dispersão dos valores no espaço, a estacionaridade intrínseca do processo pode ser corroborada por uma distribuição no espaço que não se caracteriza por uma tendência de variação dos valores com uma variação sistemática em qualquer direção. Pode-se verificar que a aplicação de água pouco modificou a distribuição espacial do ARM. Naturalmente, as regiões que receberam as maiores lâminas de água ainda apresentam valores de ARM maiores que aqueles verificados antes da aplicação. A distribuição espacial do ARM, nas Figuras 5a, b e c, permite verificar que esta distribuição depende mais de fatores intrínsecos do solo do que da distribuição da lâmina de água aplicada. Embora as características deste solo permitam caracterizá-lo como de textura uniforme, na área experimental, a variabilidade espacial da estrutura do solo pode estar ditando o padrão espacial do ARM. Nota-se que os maiores valores de ARM encontram-se nas extremidades que correspondem aos valores extremos do eixo $\mathrm{Y}$, o oposto do que ocorre com a lâmina aplicada (Figuras 5a, b, c, d). Isso revela que o padrão espacial de ARM apresenta elevada estabilidade temporal, em conformidade com o encontrado por TRINTINALHA (2005), na mesma área, corroborando a hipótese da estabilidade temporal dessa variável.

\section{CONCLUSÕES}

As variáveis porosidade total, macroporosidade e microporosidade não apresentaram estrutura de dependência espacial. A densidade do solo apresentou moderado grau de dependência espacial $(\mathrm{GD}=67,3 \%)$.

As variáveis armazenamento de água no solo e lâmina de irrigação apresentaram estrutura de dependência espacial, permitindo seu mapeamento por meio de técnicas da geoestatística.

A uniformidade de aplicação da lâmina de irrigação não alterou o padrão espacial de armazenamento de água no solo, evidenciando que fatores intrínsecos do solo definiram esse padrão. A estrutura de dependência espacial do armazenamento de água no solo apresentou o mesmo padrão com o secamento do solo.

\section{REFERÊNCIAS}

BEIGUELMAN, B. Curso prático de bioestatística. Ribeirão Preto: Sociedade Brasileira de Genética, 1994. 244 p.

BERNER, P.G.M.; VIEIRA, S.R.; LIMA, E.; ANJOS, L.H.C. Variabilidade espacial de propriedades físicas e químicas de um cambissolo sob dois sistemas de manejo de cana-de-açúcar. Revista Brasileira de Ciência do Solo, Viçosa - MG, v.31, n.5, p.837-844, 2007.

BLAKE, G.R.; HARTGE, K.H. Bulk density. In: KLUTE, A. Methods of soil analysis: Physical and mineralogical methods. Madison: America Society of Agronomy, 1986. cap.13, p.363-375.

BLAINSKI, E.; GONÇALVES, A.C.A.; TORMENA, C.A.; FOLEGATTI, M.V.; GUIMARÃES, R.M.L. Intervalo hídrico ótimo num Nitossolo Vermelho distroférrico irrigado. Revista Brasileira de Ciência do Solo, Viçosa - MG, v.33, n.2, p.273-281, 2009.

CAMBARDELLA, C.A.; MOORMAN, T.B.; NOVAK, J.M.; PARKIN, T.B.; TURCO, R.F.; KONOPKA, A.E. Field-scale variability of soil properties in central Iowa soils. Soil Science Society of America Journal, Madison, v.58, n.5, p.1.501-1.511, 1994.

CAMPOS, H. Estatística experimental não-paramétrica. Piracicaba: ESALQ, Departamento de Matemática e Estatística, 1983. 349 p.

CARVALHO, M.P.; TAKEDA E.Y.; FREDDI, O.S. Variabilidade espacial de atributos de um solo sob videira em Vitória Brasil - SP. Revista Brasileira de Ciência do Solo, Viçosa - MG, v.27, n.4, p.695-703, 2003.

CAVALIERI, K.M.V.; TORMENA, C.A.; VIDIGAL FILHO, P.S.; GONÇALVES, A.C.A.; COSTA, A.C.S. Effects of tillage systems on the soil physical properties of a dystrophic Red Latosol. Revista Brasileira de Ciência de Solo, Viçosa - MG, v.30, n.1, p.137-147, 2006 . 
CHRISTIANSEN, E.J. Irrigation by sprinkling. Berkeley: University of California, 1942. 142 p. (Bulleting, 670).

COELHO FILHO, M.A.; COELHO, R.D.; GONCALVES, A.C.A. Spatial variability of soil hydrophysical variables in a Tahiti lime orchard irrigated by micro-sprinkler. Revista Brasileira de Engenharia Agrícola e Ambiental, Campina Grande, v.5, n.2, p.239-246, 2001.

COSTA NETO, P.L.O. Estatística. São Paulo: Edgard Blücher, 1990. 264 p.

DA MATA, J.D.V.; GONÇALVES, A.C.A.; VIEIRA, S.R. Variabilidade espacial da macroporosidade do solo em área irrigada, antes do preparo e após colheita, sob dois sistemas de preparo. Acta Scientiarum, Maringá, v.20, n.3, p.307-312, 1998.

DEXTER, A.R. Advances in characterization of soil structure. Soil and Tillage Research, Amsterdam, v.11, n.1, p.199-238, 1988.

EMBRAPA. EMPRESA BRASILEIRA DE PESQUISA AGROPECUÁRIA. Centro Nacional de Pesquisa de Solos. Manual de métodos de análise de solo. 2.ed. Rio de Janeiro, 1997. 212 p.

EMBRAPA. EMPRESA BRASILEIRA DE PESQUISA AGROPECUÁRIA. Centro Nacional de Pesquisa de Solos. Sistema Brasileiro de Classificação de Solos. 2.ed. Rio de Janeiro, 2006. 306 p.

ENGLUND, E.; SPARKS, A. Geo-EAS: Geostatistical Environmental Assessmend Software. Las Vegas: U.S. Environmental Protection Agency, 1988.

FIDALSKI, J.; TORMENA, C.A. Homogeneity of soil physical quality in-between rows of an orange orchard with groundcover management systems. Revista Brasileira de Ciência do Solo, Viçosa - MG, v.31, n.4, p.637-645, 2007.

FIDALSKI, J.; TORMENA, C.A.; GONÇALVES, A.C.A.; OLIVEIRA JÚNIOR, R.S. Variabilidade espacial da resistência do solo à penetração e da taxa de estratificação de carbono orgânico do solo em um Latossolo Vermelho eutroférrico. Ciência Rural, Santa Maria, v.36, n.6, p.1.773-1.779, 2006.

FRIZZONE, J. A. Irrigação por aspersão: uniformidade e eficiência. Piracicaba: ESALQ, Departamento de Engenharia Rural, 1992. 53 p. (Série Didática, 3).

FRIZZONE, J.A.; REZENDE, R.; GONÇALVES, A.C.A.; HELBEL JÚNIOR, C. Produtividade do feijoeiro sob diferentes uniformidades de distribuição de água na superfície e na subsuperfície do solo. Engenharia Agrícola, Jaboticabal, v.27, n.2, p.414-425, 2007.

GOHRING, T.R.; WALLENDER, W.W. Economics of sprinkler irrigation systems. Transactions of the ASAE, St. Joseph, v.30, n.4, p. 1.083-1.089, 1987.

GONÇALVES, A.C.A.; FOLEGATTI, M.V. Correlação espacial entre retenção de água e textura do solo, para fins de manejo de irrigação. Engenharia Agrícola, Jaboticabal, v.22, n.3, p.288-451, 2002.

GONÇALVES, A.C.A.; FOLEGATTI, M.V.; SILVA, A.P. Estabilidade temporal da distribuição espacial da umidade do solo em área irrigada por pivô central. Revista Brasileira de Ciência do Solo, Viçosa - MG, v.23, n.1, p.155-164, 1999.

GONÇALVES, A.C.A.; TRINTINALHA, M.A.; TORMENA, C.A.; REZENDE, R.; FOLEGATTI, M.V.. Distribuição espacial do armazenamento de água no solo. In: CONGRESSO BRASILEIRO DE ENGENHARIA AGRÍCOLA, 33., 2004, São Pedro. Anais... São Pedro: Associação Brasileira de Engenharia Agrícola, 2004. 1 CD-ROM.

GRABLE, A.R.; SIEMER, E.G. Effects of bulk density, aggregate size, and soil water suction on oxygen diffusion, redox potencial and alongation of corn roots. Soil Science Society of America Journal, Madison, v.32, p.180-186, 1968. 
IAPAR. INSTITUTO AGRONÔMICO DO PARANÁ. Cartas climáticas do Paraná. Londrina: IAPAR, 2000, versão 1.0. CD-ROM.

ISAAKS, E.H.; SRIVASTAVA, R.M. An introduction to applied geostatistics. New York: Oxford University Press, 1989. $561 \mathrm{p}$.

JOURNEL, A.G.; HUIJBREGTS, Ch. J. Mining geostatistics. London: Academic Press, 1978. 600 p.

KACHANOSKI, R.G.; DE JONG, E. Scale dependence and the temporal persistence of spatial patterns of soil water storage. Water Resourses Research, Washington, v.24, n.1, p.85-91, 1988.

KHIEL, E.J. Manual de edafologia: relação solo-planta. São Paulo: Ceres, 1979. 262 p.

LETEY, J.; VAUX, H.J.; FEINERMAN, E. Optimum crop water application as affected by uniformity of water infiltration. Agronomy Journal, Madison, v. 76, n. 3, p. 435-441, 1984.

LIBARDI, P.L.; MANFRON, P.A.; MORAES, S.O.; TUON, R.L. Variabilidade da umidade gravimétrica de um solo hidromórfico. Revista Brasileira de Ciência do solo, Viçosa - MG, v.20, p.1-12, 1996.

NIELSEN, D.R.; BIGGAR, W.; ERB, K.T. Spatial variability of field-measured soil-water properties. Hilgardia, Oakland, v.42, n.7, p.215-259, 1973.

PANNATIER, Y. VarioWin: Software for Spatial Data Analysis in 2D. New York: SpringerVerlag, 1996.

PAZ, V.P.S.; FRIZZONE, J.A.; BOTREL, T.A.; FOLEGATTI, M.V. Otimização do uso da água em sistemas de irrigação por aspersão. Revista Brasileira de Engenharia Agrícola e Ambiental, Campina Grande, v.6, n.3, p.404-408, 2002.

PEEL, M.C.; FINLAYSON, B.L.; MCMAHON, T.A. Updated world map of the Köppen-Geiger climate classification. Hydrology Earth System Science, Katlenburg-Lindau, v.11, p.1.633-1.644, 2007.

REZENDE, R.; GONÇALVES, A.C.A.; FREITAS, P.S.L.; FRIZZONE, J.A.; TORMENA, C.A.; BERTONHA, A. Influência da aplicação de água na uniformidade da umidade no perfil do solo. Acta Scientiarum, Maringá, v.24, n.5, p.1.553-1.559, 2002.

ROCHA, G.C.; LIBARDI, P.L.; CARVALHO, L.A.; CRUZ, A.C.R. Estabilidade temporal da distribuição espacial da armazenagem de água em um solo cultivado com citros. Revista Brasileira de Ciência do Solo, Viçosa - MG, v.29, p.41-50, 2005.

SAS. SAS/STAT procedure guide for personal computers: version 5. Cary, 1999.

SILVA, A.P.; KAY, B.D.; PERFECT, E. Characterization of the least limiting water range. Soil Science Society America Journal, Madison, v.61, p.1.775-1.781, 1994.

SOLOMON, K.H. Yield related interpretations of irrigation uniformity and efficiency measures. Irrigation Science, Berlin, v.5, n.3, p.161-172, 1984.

SOUZA, Z.M.; MARQUES JÚNIOR, J.; PEREIRA, G.T.; BENTO, M.J.C. Variabilidade espacial de atributos físicos de um Latossolo Vermelho sob cultivo de cana-de-açúcar. Revista Brasileira de Engenharia Agrícola e Ambiental, Campina Grande, v.8, n.1, p.51-58, 2004.

STAT SOFT. Statistica for windows [eletronic manual index]. Tulsa, 1993. v. 4.3.

TORMENA, C.A. ; ARAÚJO, M.A.; FIDALSKI, J.; COSTA, J.M. Variação temporal do intervalo hídrico ótimo de um Latossolo Vermelho distroférrico em sistemas de plantio direto. Revista Brasileira de Ciência do Solo, Viçosa - MG, v.31, n.2, p.211-219, 2007.

TRINTINALHA, M.A. Utilização da TDR para avaliação da distribuição espacial e estabilidade temporal do armazenamento de água em um Nitossolo Vermelho distroférrico. 2005. $139 \mathrm{f}$. Tese (Doutorado em Produção Vegetal) - Universidade Estadual de Maringá, Maringá, 2005. 
VIEIRA, S.R.; HATFIELD, J.L.; NIELSEN, D.R.. Geostatistical theory and aplication to variability of some agronomical properties. Hilgardia, Oakland, v.51, n.3, p.1-75, 1983.

WARRICK , A.W.; NIELSEN, D.R. Spatial variability of soil physical properties in the field. In: HILLEL, D. (Ed.). Applications of soil physics. New York: Academic Press, 1980. 